\title{
Energy release due to antineutrino untrapping and diquark condensation in hot quark star evolution
}

\author{
D. N. Aguilera ${ }^{1,2}$, D. Blaschke ${ }^{1,3}$, and H. Grigorian ${ }^{1,4, \star}$ \\ 1 Fachbereich Physik, Universität Rostock, Universitätsplatz 1, 18051 Rostock, Germany \\ 2 Instituto de Física Rosario, Bv. 27 de febrero 210 bis, 2000 Rosario, Argentina \\ e-mail: deborah@darss.mpg.uni-rostock.de \\ 3 Bogoliubov Laboratory for Theoretical Physics, JINR Dubna, 141980 Dubna, Russia \\ e-mail: david@thsun1.jinr.ru \\ ${ }^{4}$ Department of Physics, Yerevan State University, Alex Manoogian Str. 1, 375025 Yerevan, Armenia \\ e-mail: hovik@darss.mpg.uni-rostock.de
}

Received 3 March 2003 / Accepted 6 November 2003

\begin{abstract}
We study the consequences of antineutrino trapping in hot quark matter for quark star configurations with possible diquark condensation. Due to the conditions of charge neutrality and $\beta$-equilibrium the flavor asymmetry increases with the number density of trapped antineutrinos. Above a critical value of the antineutrino chemical potential of $30 \mathrm{MeV}$, diquark condensation is inhibited at low densities and a two-phase structure emerges: a superconducting quark matter core surrounded by a shell of normal quark matter. When the quark star cools below a temperature $T \sim 1 \mathrm{MeV}$, the mean free path of antineutrinos becomes larger than the thickness of the normal quark matter shell so that they become suddenly untrapped. By comparing the masses of configurations with the same baryon number we estimate that the release of energy due to the antineutrino untrapping transition can be in the range of $10^{51}-10^{52} \mathrm{erg}$.
\end{abstract}

Key words. dense matter - stars: interiors - stars: evolution - stars: neutron

\section{Introduction}

The engine that drives supernova explosions and gamma ray bursts is among the most energetic phenomena in the universe and remains still puzzling (Piran \& Nakar 2002). The phase transition to a quark matter phase may be a mechanism that could release such an amount of energy (Drago \& Tambini 1999; Berezhiani et al. 2002). It has been proposed that due to the Cooper instability in dense Fermi gases, cold dense quark matter is in the color superconducting state with a nonvanishing diquark condensate (Alford et al. 2001; Blaschke et al. 2001a). The consequences of diquark condensation for the cooling of compact stars due to changes in the transport properties and neutrino emissivities have been investigated in detail, (see Blaschke et al. 2000, 2001b, 2003a; Page et al. 2000), and may even contribute to the explanation of the relatively low temperature of the pulsar in the supernova remmant 3C 58 (Grigorian et al. 2002).

Unlike the case of normal (electronic) superconductors, the pairing energy gap in quark matter is of the order of the Fermi

Send offprint requests to: D. Blaschke,

e-mail: david.blaschke@physik.uni-rostock.de

* Research supported by DFG under grant No. 436 ARM 17/1/00. energy so that diquark condensation gives considerable contributions to the equation of state (EoS), of the order of $(\Delta / \mu)^{2}$. Therefore, it has been suggested that there might be scenarios that identify the unknown source of the energy of $10^{53} \mathrm{erg}$ with a release of binding energy due to Cooper pairing of quarks in the core of a cooling protoneutron star (Hong et al. 2001). In that work the total diquark condensation energy released in a bounce of the core is estimated as $(\Delta / \mu)^{2} M_{\text {core }}$ corresponding to a few percent of a solar mass, that is $10^{52} \mathrm{erg}$. In this estimate, general relativistic effects have been disregarded. It has been shown in Blaschke et al. (2003a) by solving the selfconsistent problem of the star configurations, that these effects due to the stiffening of the EoS in the diquark condensation transition lead to an increase in the gravitational mass of the star, contrary to naive estimates.

It has also been demonstrated (Blaschke et al. 2003a) that the energy release due to cooling of a quark core in a protoneutron star does not occur by an explosive process, since the diquark condensation is a second order phase transition.

In the present work, we propose a new mechanism of energy release which involves a first order phase transition induced by antineutrino untrapping. (Anti-)neutrino trapping occurs in hot compact star configurations at temperatures 
$T \geq 1 \mathrm{MeV}$ where the mean free path of (anti-)neutrinos is smaller than the typical size of a star (Prakash et al. 2001 and references therein).

During the collapse in the hot era of protoneutron star evolution, antineutrinos are produced due to the $\beta$-processes. Since they have a small mean free path, they cannot escape and the asymmetry in the system is increased. This causes the diquark condensate to shift to higher densities or even to be inhibited depending on the fraction of trapped antineutrinos.

As the quark star cools, a two-phase structure will occur. Despite the asymmetry, the interior of the quark star (because of its large density) could consist of color superconducting quark matter, whereas in the more dilute outer shell, diquark condensation cannot occur and quark matter remains in the normal state, opaque to antineutrinos for $T \geq 1 \mathrm{MeV}$. When in the continued cooling process the antineutrino mean free path increases above the size of this normal matter shell, an outburst of neutrinos occurs and gives rise to an energy release of the order of $10^{51}-10^{52} \mathrm{erg}$. This untrapping transition is of the first order and could lead to an explosive phenomenon.

The scenario to be detailed in the present paper suggests that the first pulse of neutrinos emitted in the deleptonization stage of the core collapse, after a cooling time scale, is followed by a second pulse of antineutrinos as an observable characteristic.

\section{Hot 2SC quark matter}

The investigation of the phase structure of electrically and color neutral quark matter in $\beta$-equilibrium within quark models which assume approximate $S U(3)$ flavor symmetry has revealed (Alford \& Rajagopal 2002; Steiner et al. 2002) that the Color Flavor Locking (CFL) phase is energetically favored over the 2-Flavor Superconductivity (2SC) one. On the other hand, it has been shown (Gocke et al. 2001; Neumann et al. 2003) that at low temperatures a sequential deconfinement of light and strange quark flavors occurs in dynamical quark models that solve the three-flavor gap equations. Thus, strange quark matter phases like the CFL one appear only at high densities in the very inner core of compact stars and do not occupy a large enough volume to cause observable effects. Therefore, we consider in the present work two flavor quark matter in the 2SC phase only.

\subsection{Thermodynamic potential for asymmetric quark matter}

We consider the grand canonical thermodynamic potential for 2SC quark matter within a nonlocal chiral quark model (Blaschke et al. 2003a) where in the mean field approximation the mass gap $\phi_{f}$ and the diquark gap $\Delta$ appear as order parameters and a decomposition into color $(c \in\{r, b, g\})$ and flavor $(f \in\{u, d\})$ degrees of freedom can be made.

$\Omega_{q}\left(\left\{\phi_{f}\right\}, \Delta ;\left\{\mu_{f c}\right\}, T\right)=\sum_{c, f} \Omega^{c}\left(\phi_{f}, \Delta ; \mu_{f c}, T\right)$,

where $T$ is the temperature and $\mu_{f c}$ the chemical potential for the quark with flavor $f$ and color $c$.
The contribution of quarks with given color $c$ and flavor $f$ to the thermodynamic potential is

$\Omega^{c}\left(\phi_{f}, \Delta ; \mu_{f c}, T\right)+\Omega_{\mathrm{vac}}^{c}=\frac{\phi_{f}^{2}}{24 G_{1}}+\frac{\Delta^{2}}{24 G_{2}}$

$-\frac{1}{\pi^{2}} \int_{0}^{\infty} \mathrm{d} q q^{2}\left\{\omega\left[\epsilon_{c}\left(E_{f}(q)+\mu_{f c}\right), T\right]\right.$

$\left.+\omega\left[\epsilon_{c}\left(E_{f}(q)-\mu_{f c}\right), T\right]\right\}$,

where $G_{1}$ and $G_{2}$ are coupling constants in the scalar meson and diquark chanels, respectively. The dispersion relation for unpaired quarks with dynamical mass function $m_{f}(q)=m_{f}+$ $g(q) \phi_{f}$ is given by

$E_{f}(q)=\sqrt{q^{2}+m_{f}^{2}(q)}$.

In Eq. (2) we have introduced the notation

$\omega\left[\epsilon_{c}, T\right]=T \ln \left[1+\exp \left(-\frac{\epsilon_{c}}{T}\right)\right]+\frac{\epsilon_{c}}{2}$,

where the first argument is given by

$\epsilon_{c}(\xi)=\xi \sqrt{1+\Delta_{c}^{2} / \xi^{2}}$

When we choose the green and blue colors to be paired and the red ones to remain unpaired, we have

$\Delta_{c}=g(q) \Delta\left(\delta_{c, b}+\delta_{c, g}\right)$.

For a homogeneous system, the thermodynamic potential $\Omega_{q}$ corresponds to the pressure; therefore the constant $\Omega_{\mathrm{vac}}=$ $\sum_{c} \Omega_{\mathrm{vac}}^{c}$ is chosen such that the pressure of the physical vacuum vanishes.

The nonlocality of the interaction between the quarks is implemented via formfactor functions $g(q)$ in the momentum space. We use the Gaussian formfactor defined as

$g(q)=\exp \left(-q^{2} / \Lambda^{2}\right)$

since we could show that for this choice, stable hybrid stars are possible (Blaschke et al. 2003b). The parameters: $\Lambda=$ $1.025 \mathrm{GeV}, G_{1}=3.761 \Lambda^{2}$ and $m_{u}=m_{d}=m=2.41 \mathrm{MeV}$ are fixed by the pion mass $m_{\pi}=140 \mathrm{MeV}$, pion decay constant $f_{\pi}=93 \mathrm{MeV}$ and the constituent quark mass $m_{0}=330 \mathrm{MeV}$ at $T=\mu=0$ (Schmidt et al. 1994). The constant $G_{2}$ is a free parameter of the approach which we fix as $G_{2}=0.86 G_{1}$.

We introduce new variables: the quark chemical potential for the color $c, \mu_{q c}=\left(\mu_{u c}+\mu_{d c}\right) / 2$, and the chemical potential of the isospin asymmetry, $\mu_{\mathrm{I}}=\left(\mu_{u c}-\mu_{d c}\right) / 2$, which is color independent. We now consider symmetric $\left(\mu_{u c}=\mu_{d c}\right)$ or nearly symmetric $\left(\mu_{u c} \simeq \mu_{d c}\right)$ quark matter as the preferable situation to form a pair of fermions in the color space, i.e. $\left|\mu_{\mathrm{I}}\right|<\mu_{q c}$.

The color asymmetry induces a splitting of the quark chemical potentials relative to the mean value for two flavors $\mu_{q}$ which is proportional to the new chemical potential $\mu_{8}$. Therefore we can write,

$\mu_{q c}=\mu_{q}+\frac{\mu_{8}}{3}\left(\delta_{c, b}+\delta_{c, g}-2 \delta_{c, r}\right)$,

where $\mu_{q}$ and $\mu_{8}$ are conjugate to the quark number density and the color charge density, respectively. 
We perfom the approximation $\phi_{u}=\phi_{d}=\phi$ and express the thermodynamic potential as in Kiriyama et al. (2001)

$$
\begin{aligned}
& \Omega_{q}\left(\phi, \Delta ; \mu_{q r}, \mu_{q b}, \mu_{\mathrm{I}}, T\right)+\Omega_{\mathrm{vac}}=\frac{\phi^{2}}{4 G_{1}}+\frac{\Delta^{2}}{4 G_{2}} \\
& -\frac{1}{\pi^{2}} \int_{0}^{\infty} \mathrm{d} q q^{2}\left\{\omega\left[\epsilon_{r}\left(-\mu_{q r}-\mu_{\mathrm{I}}\right), T\right]\right. \\
& +\omega\left[\epsilon_{r}\left(\mu_{q r}-\mu_{\mathrm{I}}\right), T\right]+\omega\left[\epsilon_{r}\left(-\mu_{q r}+\mu_{\mathrm{I}}\right), T\right] \\
& \left.+\omega\left[\epsilon_{r}\left(\mu_{q r}+\mu_{\mathrm{I}}\right), T\right]\right\} \\
& -\frac{2}{\pi^{2}} \int_{0}^{\infty} \mathrm{d} q q^{2}\left\{\omega\left[\epsilon_{b}\left(E(q)-\mu_{q b}\right)-\mu_{\mathrm{I}}, T\right]\right. \\
& +\omega\left[\epsilon_{b}\left(E(q)+\mu_{q b}\right)-\mu_{\mathrm{I}}, T\right] \\
& +\omega\left[\epsilon_{b}\left(E(q)-\mu_{q b}\right)+\mu_{\mathrm{I}}, T\right] \\
& \left.+\omega\left[\epsilon_{b}\left(E(q)+\mu_{q b}\right)+\mu_{\mathrm{I}}, T\right]\right\},
\end{aligned}
$$

where the factor 2 in the last integral comes from the degeneracy of the blue and green colors $\left(\epsilon_{b}=\epsilon_{g}\right)$.

The conditions for the local extrema of $\Omega_{q}$ correspond to coupled gap equations for the two order parameters $\phi$ and $\Delta$

$$
\left.\frac{\partial \Omega}{\partial \phi}\right|_{\phi=\phi_{0}, \Delta=\Delta_{0}}=\left.\frac{\partial \Omega}{\partial \Delta}\right|_{\phi=\phi_{0}, \Delta=\Delta_{0}}=0 .
$$

The global minumum of $\Omega_{q}$ represents the state of thermodynamic equilibrium from which all equations of state can be obtained by derivation.

\subsection{Beta equilibrium with electrons and trapped (anti)neutrinos}

The stellar matter in the quark core of compact stars consists of $u$ and $d$ quarks, electrons e and antineutrinos $\bar{v}_{\mathrm{e}}$ under the conditions of

- $\beta$-equilibrium: $d \longleftrightarrow u+\mathrm{e}^{-}+\bar{v}_{\mathrm{e}}$, which in terms of chemical potentials reads

$$
\mu_{\mathrm{e}}+\mu_{\bar{v}_{\mathrm{e}}}=-2 \mu_{\mathrm{I}},
$$

charge neutrality: $\frac{2}{3} n_{u}-\frac{1}{3} n_{d}-n_{\mathrm{e}}=0$, which could also be written as

$$
n_{\mathrm{B}}+n_{\mathrm{I}}-2 n_{\mathrm{e}}=0
$$

- color neutrality: $n_{8}=\frac{2}{3} n_{q r}-\frac{1}{3} n_{q b}=0$.

Here the number densities $n_{j}$ are defined in relation to the corresponding chemical potentials $\mu_{j}$ as

$n_{j}=-\left.\frac{\partial \Omega}{\partial \mu_{j}}\right|_{\phi_{0}, \Delta_{0} ; T}$,

where the index $j$ denotes the particle species. The baryon chemical potential is defined as $\mu_{\mathrm{B}}=3 \mu_{q}-\mu_{\mathrm{I}}$. The solution of the color neutrality condition shows that $\mu_{8}$ is about 5$7 \mathrm{MeV}$ in the region of relevant densities $\left(\mu_{q} \simeq 300-500 \mathrm{MeV}\right)$. Since the isospin asymmetry is independent of $\mu_{8}$ we consider $\mu_{q c} \simeq \mu_{q}$ in our following calculations.

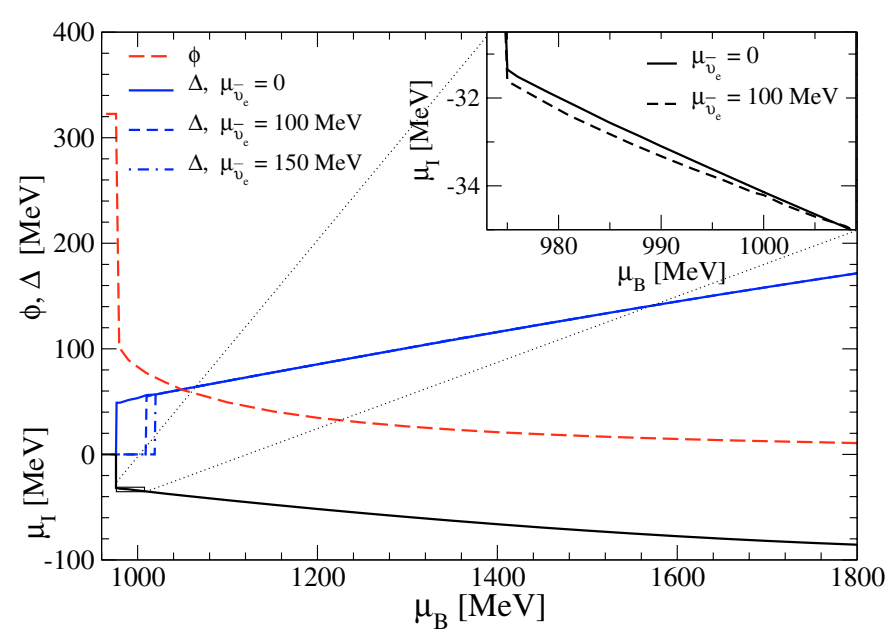

Fig. 1. Mass gap $\phi$, diquark gap $\Delta$ and isospin chemical potential $\mu_{\mathrm{I}}$ as a function of the baryon chemical potential $\mu_{\mathrm{B}}$ for different values of the antineutrino chemical potential $\mu_{\bar{\nu}_{\mathrm{e}}}$. Solutions obey $\beta$-equilibrium and charge neutrality conditions.

The total thermodynamic potential $\Omega$ contains besides the quark contribution $\Omega_{q}$ also that of the leptons $\Omega^{i d}$

$\Omega\left(\phi, \Delta ; \mu_{q}, \mu_{\mathrm{I}}, \mu_{\mathrm{e}}, \mu_{\bar{v}_{\mathrm{e}}}, T\right)=\Omega_{q}\left(\phi, \Delta ; \mu_{q}, \mu_{\mathrm{I}}, T\right)$
$+\sum_{l \in\left\{\mathrm{e}, \bar{v}_{\mathrm{e}}\right\}} \Omega^{i d}\left(\mu_{l}, T\right)$,

which is assumed to be a massless, ideal Fermi gas

$\Omega^{i d}(\mu, T)=-\frac{1}{12 \pi^{2}} \mu^{4}-\frac{1}{6} \mu^{2} T^{2}-\frac{7}{180} \pi^{2} T^{4}$.

In Fig. 1 we show the solution of the gap equations for the order parameters $\phi$ and $\Delta$ as a function of the baryon chemical potential $\mu_{\mathrm{B}}$ at $T=0$. Also the solution for the isospin chemical potential $\mu_{\mathrm{I}}$ is plotted. We can see that with increasing antineutrino chemical potential $\mu_{\bar{\nu}_{\mathrm{e}}}$ the absolute value of the chemical potential for isospin asymmetry $\left|\mu_{\mathrm{I}}\right|$ increases (see the inset of Fig. 1). We will refer to this value as the asymmetry in the system, and when it increases the onset of the superconducting phase transition is shifted to higher densities. For the region of chemical potentials $\mu_{q}$ where the diquark condensate is nonvanishing, its value coincides with that for the case $\mu_{\mathrm{I}}=0$. This region, however, shrinks for $\mu_{\bar{v}_{\mathrm{e}}}^{\min } \leq \mu_{\bar{v}_{\mathrm{e}}}$. The minimum value of antineutrino chemical potential for which the onset of diquark condensation does not coincide with the chiral transition is $\mu_{\bar{v}_{\mathrm{e}}}^{\min }=30 \mathrm{MeV}$ for a density of $\mu_{\mathrm{B}}=1 \mathrm{GeV}$.

\subsection{Equations of state}

The equations of state are obtained from the thermodynamic potential (9). For homogeneous systems, the pressure is $P=$ $-\Omega$, the entropy density is $s=-(\partial \Omega / \partial T)_{\mu_{q}}$ and the energy density is given by the Gibbs fundamental relation

$\varepsilon=s T-P+\mu_{q} n_{q}+\mu_{\mathrm{I}} n_{\mathrm{I}}$.

In Fig. 2 we show the pressure as a function of the baryon chemical potential and of the energy density for different values of the antineutrino chemical potential. The effect of the 


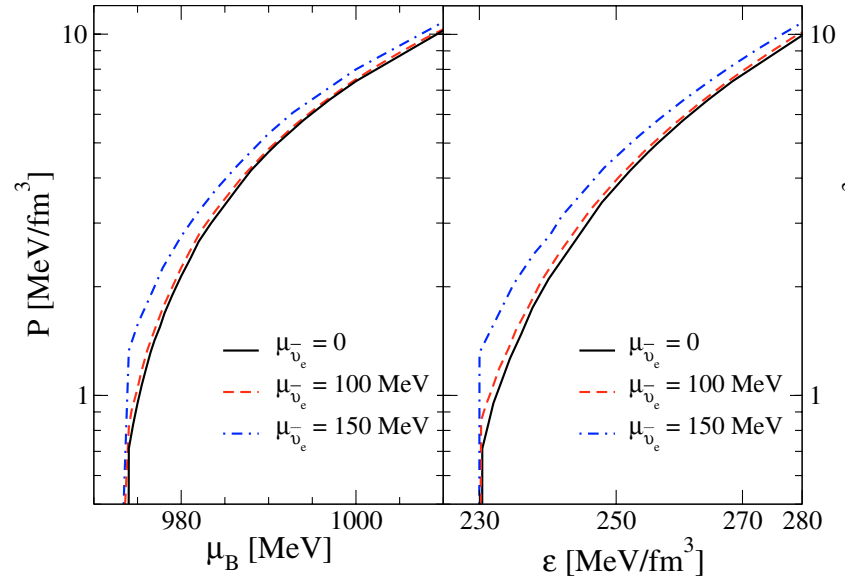

Fig. 2. Pressure vs. baryon chemical potential (left panel) and energy density (right panel) for different values of the antineutrino chemical potential $\mu_{\bar{v}_{\mathrm{e}}}$. Due to antineutrino trapping the onset of superconductivity in quark matter is shifted to higher energy densities and the equation of state becomes harder.

presence of trapped antineutrinos is twofold: (i) the onset of superconductivity in quark matter is shifted to higher energy densities with increasing $\mu_{\bar{v}_{\mathrm{e}}}$ and (ii) the equation of state becomes harder.

\section{Hot quark stars and neutrino untrapping}

Hot quark star configurations were considered first in Kettner et al. (1995) for isothermal configurations and in Blaschke et al. (1999) for adiabatic ones. When determining the mass defect of a compact star configuration due to diquark condensation in a cooling process, it has been shown in Blaschke et al. (2003a) that general relativistic effects become important. Effects that have been estimated by extrapolating the binding energy per Cooper pair (Hong et al. 2001) may even be conterbalanced when the mass distribution is determined selfconsistenly. This observation underlines the necessity to estimate the release of binding energy in evolutionary processes of compact stars which change the equation of state selfconsistently with corresponding changes in the mass distribution and the gravitational field. In Blaschke et al. (2003a) however, no trapped (anti)neutrinos have been considered and it has been demonstrated that in this case the diquark condensation occurs within a second order phase transition, so that no sudden release of energy is to be expected. The situation may change when the transition is inhibited by the presence of trapped antineutrinos which increase the asymmetry above a critical value and therefore prevent diquark condensation.

\subsection{Structure and stability of compact stars including trapped (anti)neutrinos}

We consider star configurations that are defined as solutions of the Tolman-Oppenheimer-Volkoff equations

$$
\frac{\mathrm{d} P(r)}{\mathrm{d} r}=-\frac{[\epsilon(r)+P(r)]\left[m(r)+4 \pi r^{3} P(r)\right]}{r[r-2 m(r)]},
$$

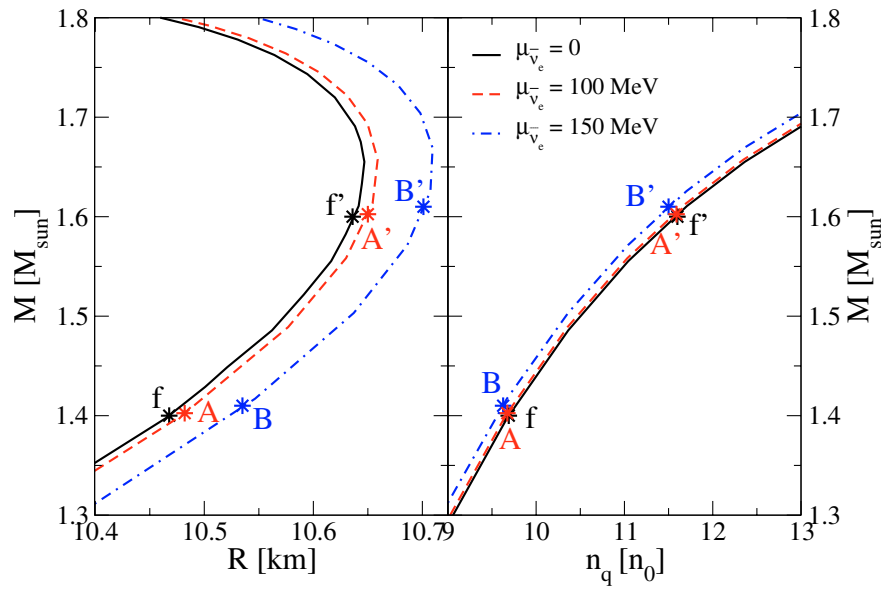

Fig. 3. Quark star configurations for different antineutrino chemical potentials $\mu_{\bar{v}_{\mathrm{e}}}=0,100,150 \mathrm{MeV}$. The total mass $M$ in solar masses ( $M_{\text {sun }} \equiv M_{\odot}$ in the text) is shown as a function of the radius $R$ (left panel) and of the central number density $n_{q}$ in units of the nuclear saturation density $n_{0}$ (right panel). Asterisks denote two different sets of configurations $(\mathrm{A}, \mathrm{B}, \mathrm{f})$ and $\left(\mathrm{A}^{\prime}, \mathrm{B}^{\prime}, \mathrm{f}^{\prime}\right)$ with a fixed total baryon number of the set.

$m(r)=4 \pi \int_{0}^{r} r^{\prime 2} \epsilon\left(r^{\prime}\right) \mathrm{d} r^{\prime}$

where $r$ is the distance from the center and $m(r)$ the mass enclosed in a sphere of radius $r$.

The equations are solved for the set of central quark number densities $n_{q}$ for which the stars are stable. The total mass $M=m(R)$ of the star is defined by the radius $R$ being the distance to the star surface which fulfills the condition of vanishing pressure $P(R)=0$.

The total mass as a function of radius and central quark number density of the configurations is plotted in Fig. 3 for the case without neutrinos $\mu_{\bar{v}_{\mathrm{e}}}=0$ (solid line) and for two cases with finite antineutrino chemical potential: $\mu_{\bar{v}_{\mathrm{e}}}=100 \mathrm{MeV}$ (dashed line) and $\mu_{\bar{v}_{\mathrm{e}}}=150 \mathrm{MeV}$ (dash-dotted line). The latter value relates to our estimate of the maximum antineutrino number density which should occur just at the deconfinement phase transition, but larger values are possible (Steiner et al. 2002). From Fig. 2 we can see that the EoS without antineutrinos is softer than that with antineutrinos (it has a lower pressure at a given energy density) and in Fig. 3 we show that this allows more compact configurations (left panel). Consequentely, the presence of antineutrinos tends to increase the mass of the star for a given central density, see the right panel of Fig. 3.

In order to estimate the effect of antineutrinos on the mass of star configurations we choose a reference configuration without antineutrinos with the mass of a typical neutron star $M_{\mathrm{f}}=1.4 M_{\odot}$, see Fig. 3. The corresponding radius is $R_{\mathrm{f}}=10.47 \mathrm{~km}$ and the central density $n_{q}=9.69 n_{0}$, where $n_{0}=0.16 \mathrm{fm}^{-3}$ is the saturation density of nuclear matter. The configurations with trapped antineutrinos and nonvanishing $\mu_{\bar{v}_{\mathrm{e}}}$ to compare with are chosen to have the same total baryon number as the reference star: $N_{\mathrm{B}}=1.51 N_{\odot}$, where $N_{\odot}$ is the total baryon number of the sun. For $\mu_{\bar{v}_{\mathrm{e}}}=100 \mathrm{MeV}$ we obtain $M_{\mathrm{A}}=1.4025 M_{\odot}$ whereas for $\mu_{\bar{v}_{\mathrm{e}}}=150 \mathrm{MeV}$ we have $M_{\mathrm{B}}=$ $1.41 M_{\odot}$. The differences in the radii are $R_{\mathrm{A}}-R_{\mathrm{f}}=0.01 \mathrm{~km}$ 
and $R_{\mathrm{B}}-R_{\mathrm{f}}=0.06 \mathrm{~km}$ and the changes in the central densities $n_{q}^{\mathrm{A}}-n_{q}^{\mathrm{f}}=-0.016 n_{0}$ and $n_{q}^{\mathrm{B}}-n_{q}^{\mathrm{f}}=-0.064 n_{0}$, respectively. This is a consequence of the hardening of the EoS due to the presence of antineutrinos. A second set of configurations ( $\mathrm{A}^{\prime}$, $\left.\mathrm{B}^{\prime}, \mathrm{f}^{\prime}\right)$ with a fixed baryon number and $M_{\mathrm{f}}=1.6 M_{\odot}$ is shown in Fig. 3.

The mass defect $\Delta M_{\mathrm{if}}=M_{\mathrm{i}}-M_{\mathrm{f}}$ can be interpreted as an energy release if there is a process which relates the configurations with $M_{\mathrm{i}}$ and $M_{\mathrm{f}}$ being the initial and final states, respectively. In the following section we discuss a possible evolution scenario and we calculate the corresponding energy release.

\subsection{Estimate of energy release in antineutrino untrapping}

The antineutrino chemical potential $\mu_{\bar{v}_{\mathrm{e}}}$ increases the asymmetry of the system and can prevent the diquark condensation provided the critical value $\mu_{\bar{v}_{\mathrm{e}}}$ is exceeded, see Fig. 1 . The inhibition of diquark condensation could provide conditions for the explosive release of energy coming from the mass defect introduced in the previous subsection.

During the collapse of a protoneutron star, neutronization takes place via the inverse $\beta$-process releasing neutrinos which can escape through the still dilute and cold outer shell of the protoneutron star. In the continuation of the collapse the temperature and the density increase in the protoneutron star core where the proton fraction rises again. Antineutrinos are created by the direct $\beta$-process in hot and dense matter and cannot escape since their mean free path is much smaller than the core radius. When under these conditions the deconfinement phase transition to quark matter occurs, this has two important consequences: (i) due to high temperatures of the order of $40 \mathrm{MeV}$ the critical density of the phase transition is much lower than at zero temperature and thus a larger fraction of the star will be in the new phase and (ii) due to antineutrino trapping the cooling is delayed.

The star cools down by surface emission of photons and antineutrinos. The region of the star where the temperature falls below the density-dependent critical value for diquark condensation will transform to the color superconducting state which is almost transparent to (anti)neutrinos. Nevertheless due to the trapped antineutrinos there is a dilute normal quark matter shell which prevents neutrino escape from the superconducting bulk of the star. The criterion for the neutrino untrapping transition is to cool the star below a temperature of about $1 \mathrm{MeV}$ when the mean free path of neutrinos becomes larger than the shell radius (Prakash et al. 2001). If at this temperature the antineutrino chemical potential is still large then the neutrinos can escape in a sudden outburst. If it is small then there will be only a gradual increase in the luminosity. An estimate for the possible release of energy within the outburst scenario can be given via the mass defect defined in the previous subsection between an initial configuration with trapped neutrinos (state A or B) and a final configuration without neutrinos (state f). In Fig. 4 the integral characteristics of the star configuration: the total mass $M$ and the total baryon number $N_{\mathrm{B}}$ are plotted as a function of the central number density $n_{q}$. We take two possible initial states

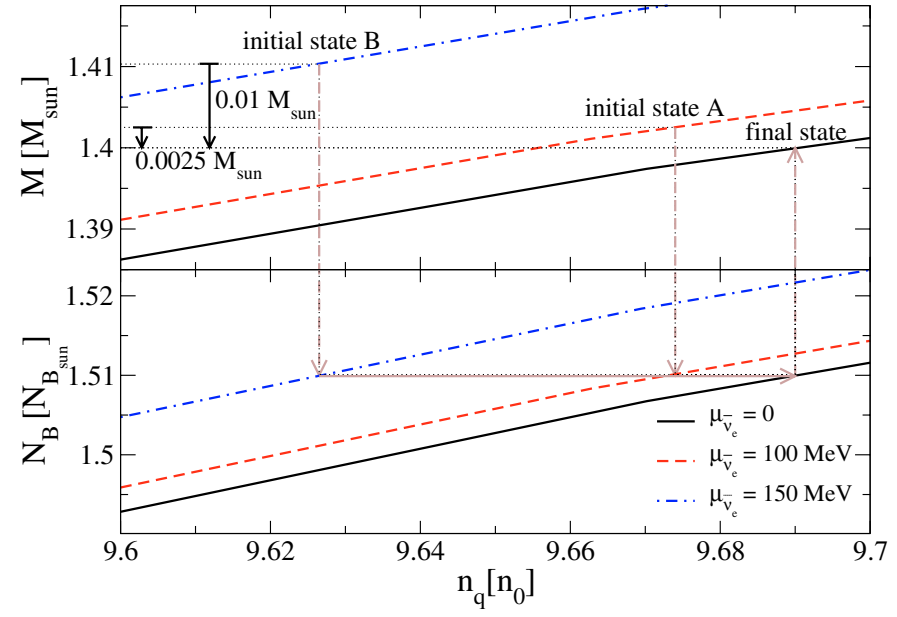

Fig. 4. Quark star configurations with diquark condensation as a function of the central number density $n_{q}$ in units of the nuclear number density $n_{0}$. The mass defect for the transition from initial configurations with $\mu_{\bar{v}_{\mathrm{e}}}=100,150 \mathrm{MeV}$ to a final configuration with $\mu_{\bar{v}_{\mathrm{e}}}=0$ at constant total baryon number is shown.

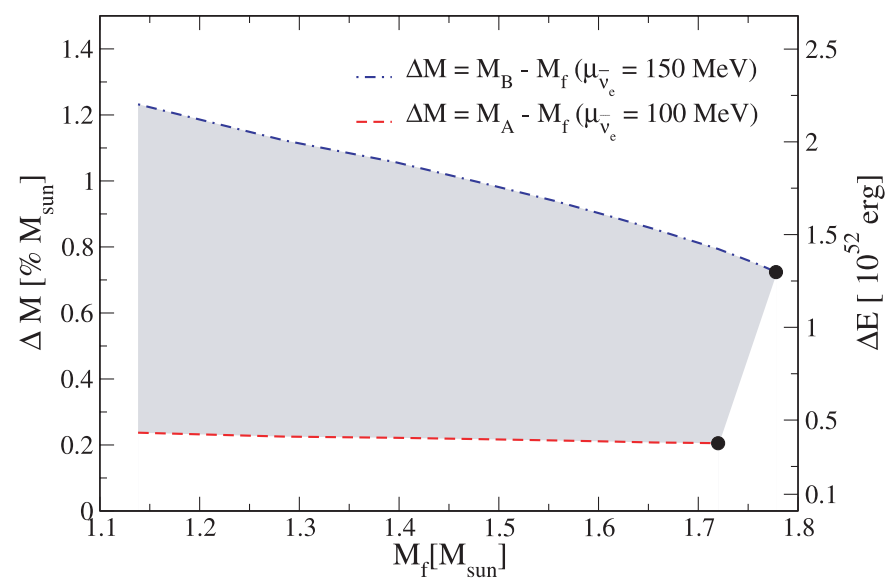

Fig. 5. Mass defect $\Delta M$ and corresponding energy release $\Delta E$ due to antineutrino untrapping as a function of the mass of the final state $M_{\mathrm{f}}$. The shaded region is defined by the estimates for the upper and lower limits of the antineutrino chemical potential in the initial state $\mu_{\bar{v}_{\mathrm{e}}}=150 \mathrm{MeV}$ (dashed-dotted line) and $\mu_{\bar{v}_{\mathrm{e}}}=100 \mathrm{MeV}$ (dashed line), respectively.

(A, B with $M_{\mathrm{A}}, M_{\mathrm{B}}$ respectively) with different antineutrino chemical potentials and we make the evolution to a final state (without antineutrinos, with $M_{\mathrm{f}}$ ) at constant total baryon number $N_{\mathrm{B}}$ as we show in Fig. 3. The configurations with trapped antineutrinos $\left(\mu_{\bar{v}_{\mathrm{e}}}=100,150 \mathrm{MeV}\right)$ correspond to possible initial states before the outburst and the configuration without antineutrinos $\left(\mu_{\bar{v}_{\mathrm{e}}}=0\right)$ is the final state after it. The mass defect ranges from $\Delta M_{\mathrm{Af}}=0.25 \% M_{\odot}$ to $\Delta M_{\mathrm{Bf}}=1 \% M_{\odot}$ for the initial states A and B, respectively. We see that the release of energy can be as large as about $1.5 \%$ of the initial mass.

We can make the same construction for different final states and in Fig. 5 we show the mass defect and the corresponding energy release as a function of the mass of the final configuration. Two lines for different initial states are plotted: the dashed one for A configurations (with $\mu_{\bar{v}_{\mathrm{e}}}=100 \mathrm{MeV}$ ) and the 
dash-dotted one for B configurations (with $\mu_{\bar{\nu}_{\mathrm{e}}}=150 \mathrm{MeV}$ ). The final state $\mathrm{f}$ with vanishing $\mu_{\bar{v}_{\mathrm{e}}}$ has the mass $M_{\mathrm{f}}$ which defines the conserved baryon number involved in the untrapping transition. The dots represent the end points of the stable configurations. The shaded region in between both curves shows the possible mass defect (or corresponding energy release) according to our estimate of the range of the antineutrino chemical potentials considered.

Since the estimated energy release is as large as $10^{51}$ $10^{52}$ erg the scenario suggested in the present paper could be discussed as a possible engine driving supernova explosions and gamma ray bursts.

A second pulse of antineutrinos is expected to be a signal of the untrapping transition.

\section{Conclusions}

We have investigated the effects of trapped antineutrinos on the asymmetry and diquark condensates in a quark star configurations. By comparing configurations with fixed baryon number the release of energy in an antineutrino untrapping transition is estimated to be of the order of $10^{52} \mathrm{erg}$. Such a transition is of the first order so that antineutrinos can be released in a sudden process (burst). This scenario could play an important rôle in solving the problem of the engine of supernova explosions and gamma ray bursts. A second antineutrino pulse is suggested as an observable characteristic of the present scenario.

Acknowledgements. Research of D.N.A. was supported in part by the CONICET PIP 03072 (Argentina), by the DFG GK 567 "Stark korrelierte Vielteilchensysteme" (Rostock University), by DAAD grant No. A/01/17862 and by Landesgraduiertenförderung von Mecklenburg Vorpommern (Germany). H.G. acknowledges support by DFG under grant No. 436 ARM 17/5/01. D.N.A. and H.G. acknowledge the hospitality of the Department of Physics at the University of Rostock where this research has been performed.

\section{References}

Alford, M., \& Rajagopal, K. 2002, JHEP, 06, 031

Alford, M. G., Bowers, J. A., \& Rajagopal, K. 2001, J. Phys., G27, 541

Berezhiani, Z., Bombaci, I., Drago, A., Frontera, F., \& Lavagno, A. 2002, Nucl. Phys. Proc. Suppl., 113, 268

Blaschke, D., Fredriksson, S., Grigorian, H., \& Oztas, A. M. 2003a [nucl-th/0301002]

Blaschke, D., Glendenning, N. K., \& Sedrakian, A. 2001a, Lecture Notes Phys., 578

Blaschke, D., Grigorian, H., Aguilera, D. N., Yasui, S., \& Toki, H. 2003b, AIP Conf. Proc., 660, 209

Blaschke, D., Grigorian, H., Poghosyan, G., Roberts, C. D., \& Schmidt, S. M. 1999, Phys. Lett. B, 450, 207

Blaschke, D., Grigorian, H., \& Voskresensky, D. N. 2001b, A\&A, 368, 561

Blaschke, D., Klahn, T., \& Voskresensky, D. N. 2000, ApJ, 533, 406

Drago, A., \& Tambini, U. 1999, J. Phys. G, 25, 971

Gocke, C., Blaschke, D., Khalatyan, A., \& Grigorian, H. 2001, Exploring Quark Matter Proc. (Rostock University Press), 239 [hep-ph/0104183]

Grigorian, H., Blaschke, D., \& Voskresensky, D. N. 2002, MPG-VTUR 231/02

Hong, D. K., Hsu, S. D. H., \& Sannino, F. 2001, Phys. Lett. B, 516, 362

Kettner, C., Weber, F., Weigel, M. K., \& Glendenning, N. K. 1995, Phys. Rev. D, 51, 1440

Kiriyama, O., Yasui, S., \& Toki, H. 2001, Int. J. Mod. Phys. E, 10, 501

Neumann, F., Buballa, M., \& Oertel, M. 2003, Nucl. Phys. A, 714, 481

Page, D., Prakash, M., Lattimer, J. M., \& Steiner, A. 2000, Phys. Rev. Lett., 85, 2048

Piran, T., \& Nakar, E. 2002, eConf, C010815, 182

Prakash, M., Lattimer, J. M., Sawyer, R. F., \& Volkas, R. R. 2001, Ann. Rev. Nucl. Part. Sci., 51, 295

Schmidt, S. M., Blaschke, D., \& Kalinovsky, Y. L. 1994, Phys. Rev. C, 50,435

Steiner, A. W., Reddy, S., \& Prakash, M. 2002, Phys. Rev. D, 66, 094007 